\title{
Local Granger causality
}

\author{
Sebastiano Stramaglia $\odot,{ }^{1}$ Tomas Scagliarini $\odot,{ }^{1}$ Yuri Antonacci $\odot,{ }^{2}$ and Luca Faes ${ }^{3}$ \\ ${ }^{1}$ Dipartimento Interateneo di Fisica, Universitá degli Studi di Bari Aldo Moro, and INFN, Sezione di Bari, 70126 Bari, Italy \\ ${ }^{2}$ Dipartimento di Fisica e Chimica, Universitá di Palermo, 90123 Palermo, Italy \\ ${ }^{3}$ Dipartimento di Ingegneria, Universitá di Palermo, 90128 Palermo, Italy
}

(Received 28 October 2020; revised 2 January 2021; accepted 26 January 2021; published 11 February 2021)

\begin{abstract}
Granger causality (GC) is a statistical notion of causal influence based on prediction via linear vector autoregression. For Gaussian variables it is equivalent to transfer entropy, an information-theoretic measure of time-directed information transfer between jointly dependent processes. We exploit such equivalence and calculate exactly the local Granger causality, i.e., the profile of the information transferred from the driver to the target process at each discrete time point; in this frame, GC is the average of its local version. We show that the variability of the local GC around its mean relates to the interplay between driver and innovation (autoregressive noise) processes, and it may reveal transient instances of information transfer not detectable from its average values. Our approach offers a robust and computationally fast method to follow the information transfer along the time history of linear stochastic processes, as well as of nonlinear complex systems studied in the Gaussian approximation.
\end{abstract}

DOI: 10.1103/PhysRevE.103.L020102

Granger causality (GC) [1] and its nonparametric counterpart, namely transfer entropy (TE) [2], are widely used tools to detect and quantify causal relationships between stochastic processes mapping the evolution of coupled dynamic systems over time. For discrete-time stationary multivariate processes represented by vector autoregressive (VAR) models [3], GC measures the gain in the linear predictability of the target process when the knowledge of the candidate driver process is exploited to make the prediction. For Gaussian systems, GC and TE are equivalent [4] and are interpreted as measures of information transfer [5].

The question we address here is as follows: is it possible to calculate the temporal profile of the information transfer in complex systems, so that its time-average coincides with the information-theoretic value of GC?

Concerning TE, the same question has been addressed in [6] with the introduction of the local transfer entropy. Differently from the corresponding averaged quantity, the local transfer entropy can be both positive and negative: when it is negative at a given time step, the observation of the driver is misinformative about the value of the target at that time. Recently, the local TE has been proposed to study phase-amplitude coupling in electrophysiological signals [7]. In our opinion, since its inception the local TE has been used in a quite limited way with respect to its potentiality: the lack of benchmark systems with an exact solution, as well as critical choices (parameters, embedding schemes) that influence the estimation of local TE, have certainly limited the popularity of this notion. In this work, we show that it is possible to calculate exactly the local Granger causality $L_{\mathrm{gc}}$ from the parameters of the underlying VAR model. Knowledge of the exact value of the local TE in benchmark systems is helpful to get the correct interpretation of the local information transfer, with particular regard to of its negative values.

Given $n$ zero-mean processes $x_{1}, \ldots, x_{n}$, we model them by a VAR model of order $p$ which, under suitable conditions [3], is assumed to be stable. This implies that the system is stationary and ergodic with time-invariant variances and covariances. The past of the system at time $t$ is described by the vector $\mathbf{x}_{t}=\left[x_{1, t-1} \cdots x_{n, t-1} x_{1, t-2} \cdots x_{n, t-2} \cdots x_{n, t-p}\right]^{T}$, whose distribution is a multivariate Gaussian in the stationary regime. Calling $\beta$ and $\alpha$ the indices of the driving and target variables, respectively, we denote $y_{t}=x_{\alpha, t}$ the present state of the target, $\mathbf{w}_{t}=\left[x_{\beta, t-1} \cdots x_{\beta, t-p}\right]^{T}$ the vector of the driver's past variables, and $\mathbf{u}_{t}=\mathbf{x}_{t} \backslash \mathbf{w}_{t}$ the remaining past variables. After calculation of the conditional probabilities $p\left(y_{t} \mid \mathbf{u}_{t}, \mathbf{w}_{t}\right)$ and $p\left(y_{t} \mid \mathbf{u}_{t}\right)$, we evaluate the local Granger causality and obtain the following structure:

$$
L_{\mathrm{gc}}\left(\mathbf{u}_{t}, \mathbf{w}_{t}, y_{t}\right)=\mathrm{GC}+\mathcal{L}\left(\mathbf{u}_{\mathbf{t}}, \mathbf{w}_{\mathbf{t}}, y_{t}\right),
$$

where GC is a constant coinciding with the standard definition of Granger causality, while $\mathcal{L}$ is a function of $\mathbf{u}_{t}, \mathbf{w}_{t}, y_{t}$. Specifically, the two terms in (1) can be expressed from the second-order statistics of the processes as

$$
\mathrm{GC}=\ln \frac{\left|\boldsymbol{\Sigma}_{\mathbf{u w}}\right|\left|\boldsymbol{\Sigma}_{y \mathbf{u}}\right|}{\left|\boldsymbol{\Sigma}_{y \mathbf{u w}}\right|\left|\boldsymbol{\Sigma}_{\mathbf{u}}\right|},
$$

where $|\cdot|$ stands for the determinant of a matrix, and

$$
\begin{aligned}
\mathcal{L}\left(\mathbf{u}_{t}, \mathbf{w}_{t}, y_{t}\right)= & \mathbf{Z}_{\mathbf{u w}, t}^{T} \boldsymbol{\Sigma}_{\mathbf{u w}}^{-1} \mathbf{Z}_{\mathbf{u w}, t}+\mathbf{Z}_{y \mathbf{u}, t}^{T} \boldsymbol{\Sigma}_{y \mathbf{u}}^{-1} \mathbf{Z}_{y \mathbf{u}, t} \\
& -\mathbf{Z}_{y \mathbf{u w}, t}^{T} \boldsymbol{\Sigma}_{y \mathbf{u w}}^{-1} \mathbf{Z}_{y \mathbf{u w}, t}-\mathbf{u}_{t}^{T} \boldsymbol{\Sigma}_{\mathbf{u}}^{-1} \mathbf{u}_{t},
\end{aligned}
$$

where $\quad \mathbf{Z}_{\mathbf{u w}, t}=\left[\mathbf{u}_{t}^{T} \mathbf{w}_{t}^{T}\right]^{T}, \quad \mathbf{Z}_{y \mathbf{u}, t}=\left[y_{t} \mathbf{u}_{t}^{T}\right]^{T}, \quad$ and $\quad \mathbf{Z}_{y \mathbf{u w}, t}=$ $\left[y_{t} \mathbf{u}_{t}^{T} \mathbf{w}_{t}^{T}\right]^{T}$ are the observations of the present and past 


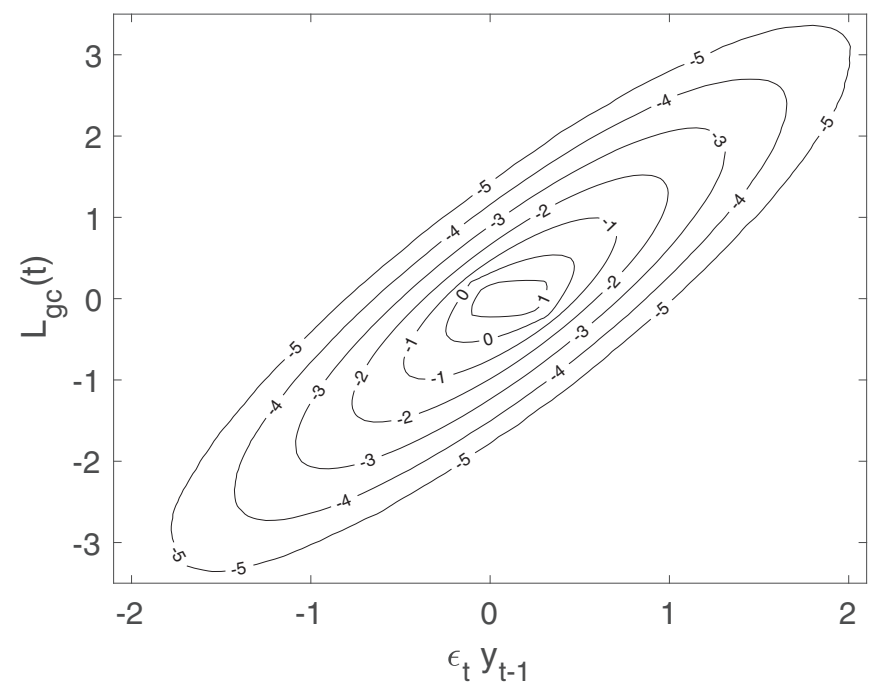

FIG. 1. Distribution density of the pairs $\left[\epsilon_{t} y_{t-1}, L_{\mathrm{gc}}(t)\right]$ sampled from the time evolution of model (4) depicted as a contour plot. Values displayed on the level curves are the logarithm of the corresponding value of the distribution density.

states of the processes, and $\boldsymbol{\Sigma}_{\mathbf{u w}}=\mathbb{E}\left[\mathbf{Z}_{\mathbf{u w}, t} \mathbf{Z}_{\mathbf{u w}, t}^{T}\right], \quad \boldsymbol{\Sigma}_{\mathbf{y u}}=$ $\mathbb{E}\left[\mathbf{Z}_{y \mathbf{u}, t} \mathbf{Z}_{y \mathbf{u}, t}^{T}\right], \boldsymbol{\Sigma}_{y \mathbf{u w}}=\mathbb{E}\left[\mathbf{Z}_{y \mathbf{u w}, t} \mathbf{Z}_{y \mathbf{u w}, t}^{T}\right]$, and $\boldsymbol{\Sigma}_{\mathbf{u}}=\mathbb{E}\left[\mathbf{u}_{t} \mathbf{u}_{t}^{T}\right]$ are the relevant covariance matrices. Importantly, $\mathcal{L}$ has a vanishing expected value, therefore $\left\langle L_{\mathrm{gc}}(t)\right\rangle=\mathrm{GC}$. The derivation of the local GC in (1) from the parameters of the VAR model fitting the observed processes is reported in the Supplemental Material [8].

To characterize negative values and temporal profiles of the local Granger causality, we consider the following simple toy model:

$$
\begin{aligned}
& y_{t}=\tilde{\epsilon}_{t}, \\
& x_{t}=0.2 x_{t-1}+0.4 y_{t-1}+\epsilon_{t},
\end{aligned}
$$

where $\tilde{\epsilon}$ and $\epsilon$ are white noise terms with standard deviation $\sigma_{\tilde{\epsilon}}=1$ and $\sigma_{\epsilon}=0.8$. The GC $y \rightarrow x$ is $\mathrm{GC}=0.18$ in this case, corresponding to the mean of the local quantity $L_{\mathrm{gc}}(t)$. In Fig. 1, we depict the distribution of sample points in the plane $\left(\epsilon_{t} y_{t-1}\right)-L_{\mathrm{gc}}(t)$, obtained from a run of (4) with length $30 \times 10^{6}$ time steps. The plot shows that the local GC oscillates between positive and negative values, attaining large negative values when $\epsilon_{t} y_{t-1}$ is large and negative. The latter situation occurs when the noise pulls the system in the opposite direction with respect to the action of the cause $y_{t-1}$ : in this case, the knowledge of $y_{t-1}$ is misinformative about $x_{t}$, meaning that a reduced model implemented without using the driver performs better than the full model in (4). Conversely, large positive values are attained at times $t$ when the noise term $\epsilon_{t}$ pulls the system in the same direction as the cause $y_{t-1}$. It is worth stressing, therefore, that fluctuations of $L_{\mathrm{gc}}$ do not merely reproduce modulations of the noise of the system, but rather they represent the interplay between noise and the driving variable. These fluctuations constitute, in addition to their mean value, a hallmark of information transfer, as it

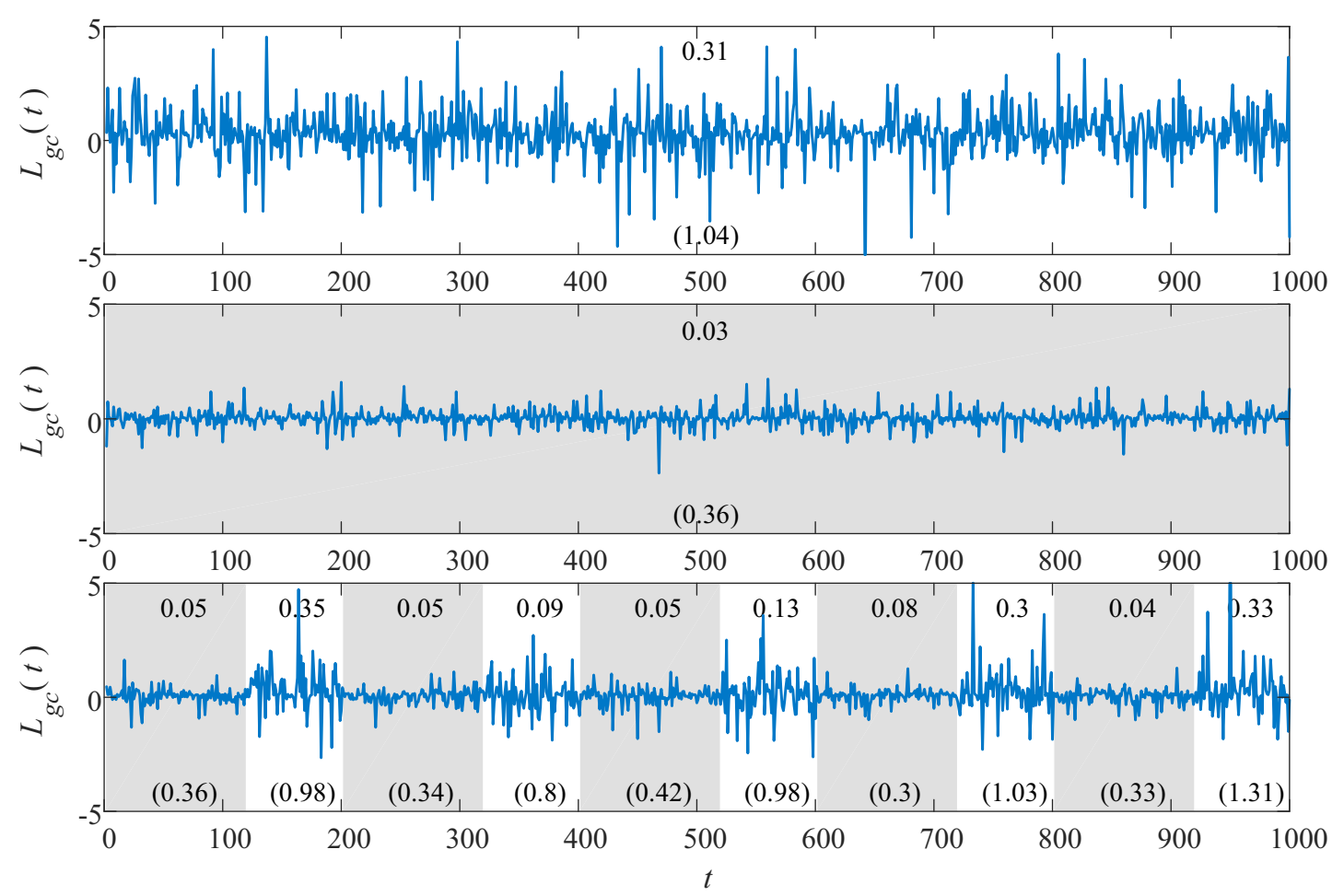

FIG. 2. Time course of the local GC computed for the toy model (4) along the direction $y \rightarrow x$ under different values of the standard deviation of the driving variable $y$, i.e., $\sigma_{\tilde{\epsilon}}^{2}=2$ (top), $\sigma_{\tilde{\epsilon}}^{2}=0.2$ (middle), and $\sigma_{\tilde{\epsilon}}^{2}$ alternating between 0.2 and 2 at the time points $t \in\{120,200,320,400,520,600,720,800,920\}$ (bottom). The mean and standard deviation of the $L_{\mathrm{gc}}$ computed within each time window where $\sigma_{\tilde{\epsilon}}^{2}=2$ (gray shaded epochs), or where $\sigma_{\tilde{\epsilon}}^{2}=0.2$ (white epochs), are reported, respectively, at the top and at the bottom (in brackets) of the window. 

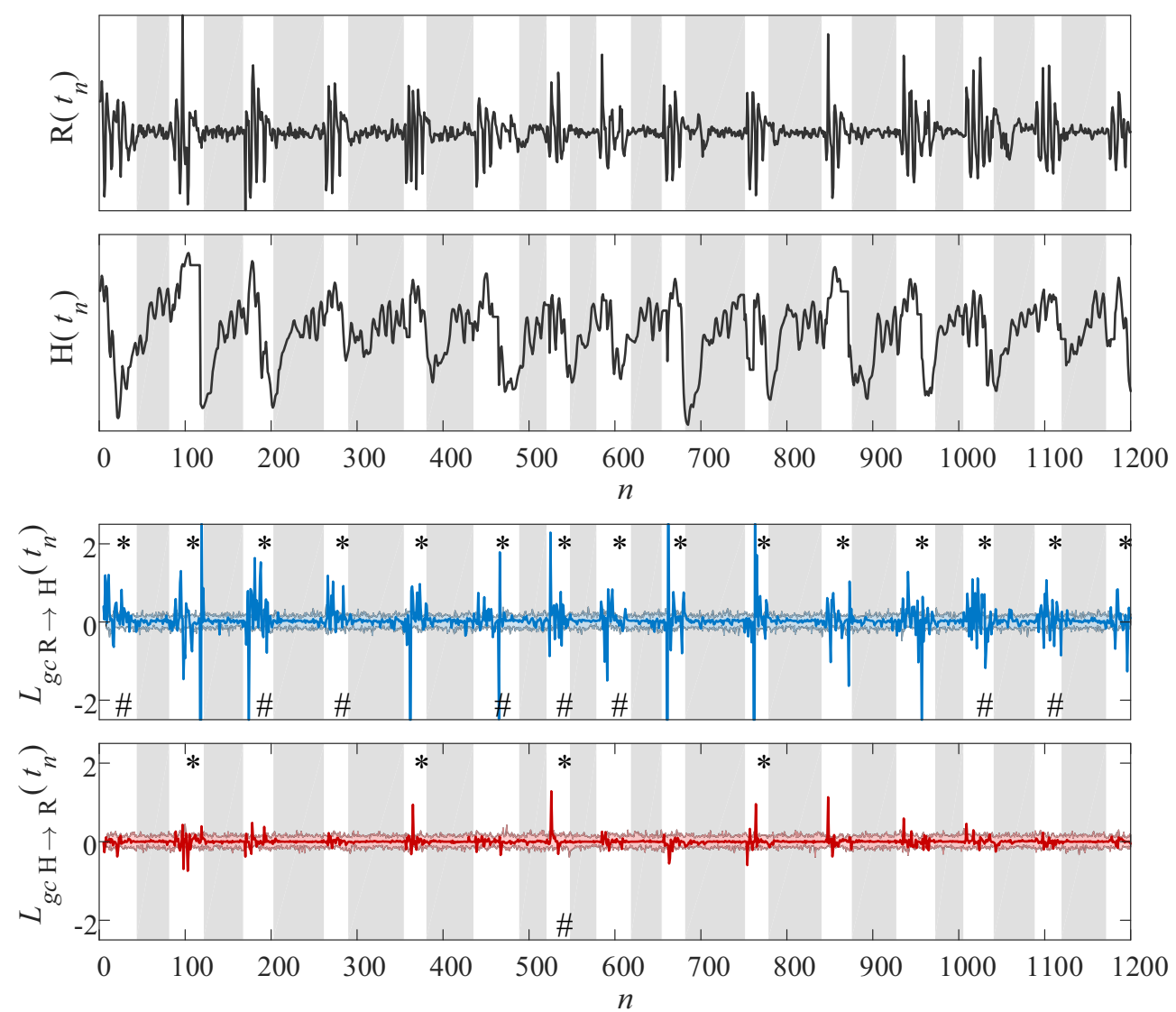

FIG. 3. Time series of respiration $\left[R\left(t_{n}\right)\right]$ and heart rate $\left[H\left(t_{n}\right)\right]$ measured for a subject exhibiting several instances of sleep apneas (gray shades in the plots), and corresponding time courses of the local GC computed from $\mathrm{R}$ to $\mathrm{H}\left[L_{\mathrm{gc} R \rightarrow H}\left(t_{n}\right)\right]$ and from $\mathrm{H}$ to $\mathrm{R}\left[L_{\mathrm{gc} H \rightarrow R}\left(t_{n}\right)\right]$. The discrete time points are $t_{n}=n \Delta T$, with $\Delta T=0.5 \mathrm{~s}$. The local GC courses are plotted together with the corresponding significance bounds (horizontal colored shades) obtained from IAAFT surrogates [11]. The symbols \# and $*$ mark statistically significant values of the mean and standard deviation of the local GC computed within each apneic or nonapneic time window (gray and white areas).

can be seen in Fig. 2, where the local GC $y \rightarrow x$ is reported for different runs of the simulation performed changing the variance of the driving variable $y$ : we find that not only the mean, but also the amplitude of the oscillations of the local GC is modulated by the strength $\sigma_{y}=\sigma_{\tilde{\epsilon}}$ of the driving variable. For small $\sigma_{y}$, in this toy model we find $\mathrm{GC}=\left\langle L_{\mathrm{gc}}\right\rangle \sim \sigma_{y}^{2}$ and $\left\langle L_{\mathrm{gc}}^{2}\right\rangle-\left\langle L_{\mathrm{gc}}\right\rangle^{2} \sim \sigma_{y}^{2}$.

As a first application example, we take the bivariate time series of respiration $(R)$ and heart rate $(H)$ amplitudes measured with a sampling rate of $2 \mathrm{~Hz}$ from a subject suffering from sleep apneas and previously analyzed with local TE [2] and nonlinear GC [9]. Figure 3 shows that consecutive apneas are characterized by the absence of respiratory oscillations and progressively increasing heart rate. Adopting the Gaussian approximation, these time series are fitted with a bivariate autoregressive model of order 4 , identified with the Akaike Information criterion [10]. Then, we compute both the global and local GC along the two directions of interaction, as well as their significance thresholds based on iterative amplitude-adjusted Fourier transform (IAAFT) surrogates [11]. The GC is statistically significant along the direction from respiration to heart rate $\left(\mathrm{GC}_{R \rightarrow H}=0.0341\right.$, IAAFT 95th percentile $=0.0096$ ), while it is low and nonsignificant along the opposite direction $\left(\mathrm{GC}_{H \rightarrow R}=0.0015\right.$, IAAFT 95th percentile $=0.0079$ ); physiologically, this result supports the mainly unidirectional nature of respiratory sinus arrhythmia [12]. Computation of the local GC supports the lack of interactions from heart rate to respiration, and reveals the local nature of the information transfer from respiration to heart rate: the $L_{\mathrm{gc}} R \rightarrow H$ exhibits clear marked oscillations with statistically significant mean and standard deviation only while the patient is breathing, while it is very small and nonsignificant during the apneas. It is worth mentioning that the absence of significant GC during the apneas and the unidirectional GC $R \rightarrow H$ in the nonapneic state were observed also fitting the VAR model over the time windows corresponding to the two states. Nevertheless, we remark that our approach, sharing the philosophy of local information dynamics [6,13], differs fundamentally from time-varying GC methods [14]; these methods fit a different VAR model on the data from each predefined temporal window, requiring stationarity within each window and being subject to the tradeoff between estimation accuracy and temporal resolution. On the contrary, local methods like ours fit the model on the whole time series globally assumed as stationary, and then provide a pointwise measure without the need of identifying and sizing the analysis windows.

As another real example, we consider the analysis of signals from an epileptic brain. Although significant information can be extracted from electroencephalogram (EEG) 

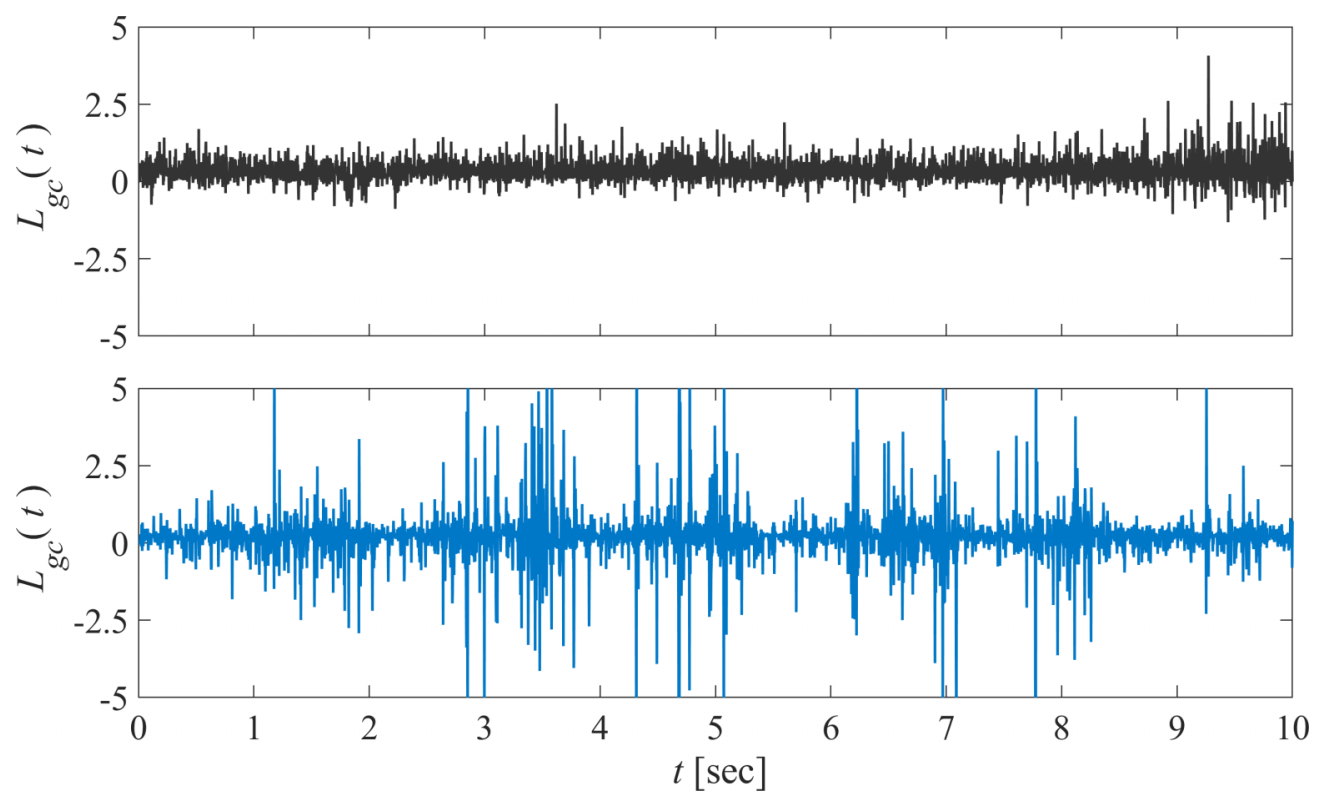

FIG. 4. Time course of the local GC computed from the deep electrode to the cortical electrodes (average over 64 target electrodes) computed in the pre-ictal stage (black, upper panel) and in the ictal stage (blue, lower panel).

recordings made from the scalp surface of patients, in some cases of pharmaco-resistant epilepsy (when medications fail to prevent seizures), invasive electrocorticogram (ECoG) recordings are performed, with recordings of voltage directly from the brain's surface or deep brain regions. Here we consider ECoG recordings from a drug-resistant epilepsy patient from 64 cortical electrodes (placed with $8 \times 8$ geometry) and two depth electrodes with six contacts each [15], available at [16]. Many studies of transfer entropy in the epileptic brain are published; see, e.g., [17]. Here we analyze these signals in the Gaussian approximation. Data are sampled at $400 \mathrm{~Hz}$, and we apply the proposed method on the fourth seizure, considering two 10-s windows in the pre-ictal stage (before the epileptic seizure) and in the ictal stage (during the seizure). A previous paper [18] showed that one of the depth electrodes is close to the seizure onset zone, i.e., the site of the beginning of the epileptic seizures and of their primary organization. Therefore, we evaluate in a pairwise fashion the local Granger causality from that depth electrode to all 64 cortical electrodes. Data are fitted with a VAR with order $p$ chosen according to Akaike's criterion [10]: our results, averaged over the cortical targets, are displayed in Fig. 4. In the pre-ictal stage, averaging over the targets leads to a homogeneous pattern, with $\mathrm{GC}=0.32$; on the other hand, in the ictal stage after averaging, the mean $\mathrm{GC}$ is lower $(\mathrm{GC}=$ 0.23 ) but the temporal profile is more intermittent and shows peaks of $L_{\mathrm{gc}}$ in correspondence with time instants in which the source coherently transmits information to a large portion of the cortical electrodes. These results show, on the one hand, that as expected the pattern of the information flow in the epileptic brain is different before and during the seizure. On the other hand, it clearly suggests that the classical measure of Granger causality (the mean of $L_{\mathrm{gc}}$ ) is not sufficient to properly describe the temporal properties of the information transfer in this system; for example, contrary to the mean $L_{\mathrm{gc}}$, the standard deviation of $L_{\mathrm{gc}}$ increases from 0.36 in the pre-ictal stage to 0.89 during the seizure. Therefore, the standard deviation of $L_{\mathrm{gc}}$ conveys in this case a description of the pattern of information transfer complementary to that provided by the standard GC.

Both the simulation example and the two applications have revealed the important role of the variability displayed by the proposed local GC measure around its mean. In fact, our results indicate that the local GC may exhibit fluctuations of varying magnitude in correspondence with local modifications of the process dynamics: we have reported instances of locally increased variance of the $L_{\mathrm{gc}}$ profiles which can or cannot be associated with increased mean values representative of higher GC (as happens, e.g., in the two reported applications). The modulation of $L_{\mathrm{gc}}$ fluctuations may thus reveal the transient instances of information transfer in the system.

Summarizing, local Granger causality implements the notion of local transfer entropy for linear systems, and it can be used to extract the temporal profile of information transfer; we have derived its expression for a generic VAR model. As fitting a VAR model to data only requires the choice of the order $p$, our formalism can be easily used to extract the temporal profile of information transfer for linear systems. We remark that in many applications, nonlinearities can be neglected and the Gaussian approximation fully captures the underlying phenomena; if this is not the case, the results obtained in the Gaussian approximation still constitute the reference to which one should refer for assessing the role of nonlinearities. Moreover, we have illustrated the usefulness of the local GC also in the presence of transient alterations of the process dynamics inducing departure from Gaussianity. We have shown that fluctuations of $L_{\mathrm{gc}}$ are connected to the interplay between the innovation (noise) and driver processes, in such a way that large negative (positive) values correspond to the noise pulling the system in the opposite (same) direction as the driver. Given that innovations model the environment 
acting on the system under consideration, and in agreement with the discussion in [5], we conclude that negative (misinformative) values of $L_{\mathrm{gc}}$ are important as they are the signature of extra features in the dynamics that have not been accounted for in the past of the measured variables alone. Since GC has gained increasing popularity in many fields of science, we

[1] C. W. J. Granger, J. Econom. Soc. 37, 424 (1969).

[2] T. Schreiber, Phys. Rev. Lett. 85, 461 (2000).

[3] J. D. Hamilton, Time Series Analysis (Princeton University Press, Princeton, NJ, 1994).

[4] L. Barnett, A. B. Barrett, and A. K. Seth, Phys. Rev. Lett. 103, 238701 (2009).

[5] T. Bossomaier, L. Barnett, M. Harré, and J. T. Lizier, An Introduction to Transfer Entropy (Springer, Cham, Switzerland, 2016).

[6] J. T. Lizier, M. Prokopenko, and A. Y. Zomaya, Phys. Rev. E 77, 026110 (2008).

[7] R. Martínez-Cancino, A. Delorme, J. Wagner, K. KreutzDelgado, R. C. Sotero, and S. Makeig, Entropy 22, 1262 (2020).

[8] See Supplemental Material at http://link.aps.org/supplemental/ 10.1103/PhysRevE.103.L020102 for a derivation of the local GC in Eq. (1) from the parameters of the VAR model. expect that the proposed approach will have a large impact as it allows us to estimate easily the information transfer during the time evolution of a complex system.

This research was supported by MIUR project PRIN 2017WZFTZP "Stochastic forecasting in complex systems."
[9] D. Marinazzo, M. Pellicoro, and S. Stramaglia, Phys. Rev. Lett. 100, 144103 (2008).

[10] A. Akaike, IEEE Trans. Aut. Control 19, 716 (1974).

[11] T. Schreiber and A. Schmitz, Phys. Rev. Lett. 77, 635 (1996).

[12] F. Yasuma, J. Hayano Chest 125, 683 (2004).

[13] J. T. Lizier, The Local Information Dynamics of Distributed Computation in Complex Systems (Springer Science, Berlin, 2012).

[14] W. Hesse, E. Moller, M. Arnold, and B. Schack, J. Neurosci. Methods 124, 27 (2003).

[15] M. A. Kramer, E. D. Kolaczyk, and H. E. Kirsch, Epilepsy Res. 79, 173 (2008).

[16] http://math.bu.edu/people/kolaczyk/datasets.html

[17] M. Staniek and K. Lehnertz, Phys. Rev. Lett. 100, 158101 (2008).

[18] L. Faes, D. Marinazzo, and S. Stramaglia, Entropy 19, 408 (2017). 